\title{
Malrotated sinus vortices in straight graft valve-sparing aortic root treatment: A matter of concern?
}

\author{
Thekla H. Oechtering, MD, ${ }^{\mathrm{a}}$ Alex Frydrychowicz, MD, ${ }^{\mathrm{a}}$ and Hans-Hinrich Sievers, $\mathrm{MD}^{\mathrm{b}}$
}

\footnotetext{
From the a Clinic for Radiology and Nuclear Medicine and ${ }^{\mathrm{b}}$ Department of Cardiac and Thoracic Vascular Surgery, University Hospital Schleswig-Holstein, Lübeck, Germany.

Disclosures: H-H.S. receives royalties from B. Braun Melsungen AG. All other authors have nothing to disclose with regard to commercial support.

Received for publication Nov 22, 2016; revisions received Jan 26, 2017; accepted for publication Feb 14, 2017; available ahead of print March 20, 2017.

Address for reprints: Thekla H. Oechtering, MD, Clinic for Radiology and Nuclear Medicine, University Hospital Schleswig-Holstein, Campus Lübeck, Ratzeburger Allee 160, Lübeck 23562, Germany (E-mail: Thekla. Oechtering@uksh.de).

J Thorac Cardiovasc Surg 2017;154:794-7

$0022-5223 / \$ 36.00$

Copyright (c) 2017 by The American Association for Thoracic Surgery

http://dx.doi.org/10.1016/j.jtcvs.2017.02.024
}

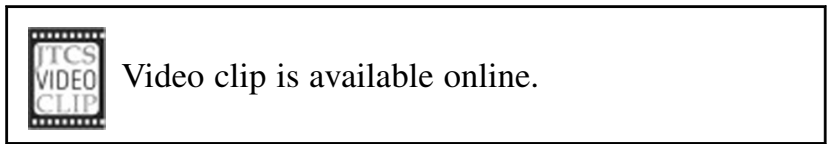

So-called valve-sparing aortic root replacement techniques $^{1}$ allow for aneurysm repair without aortic valve replacement, but do they really "spare" the aortic valve? The replacement of the aortic root has extensive

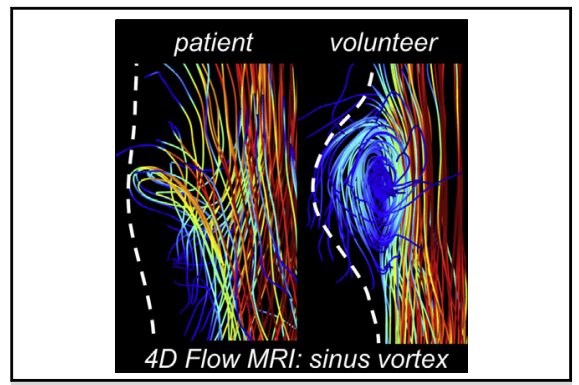

Malrotated patient's vortex after straight graft David procedure compared with a volunteer.

\section{Central Message}

Compared with healthy volunteers, sinus vortices in patients after the straight graft David procedure were rotated $90^{\circ}$ along the vessel axis. This may have yet unknown consequences for aortic valve function.

See Editorial Commentary page 798.

See Editorial page 791

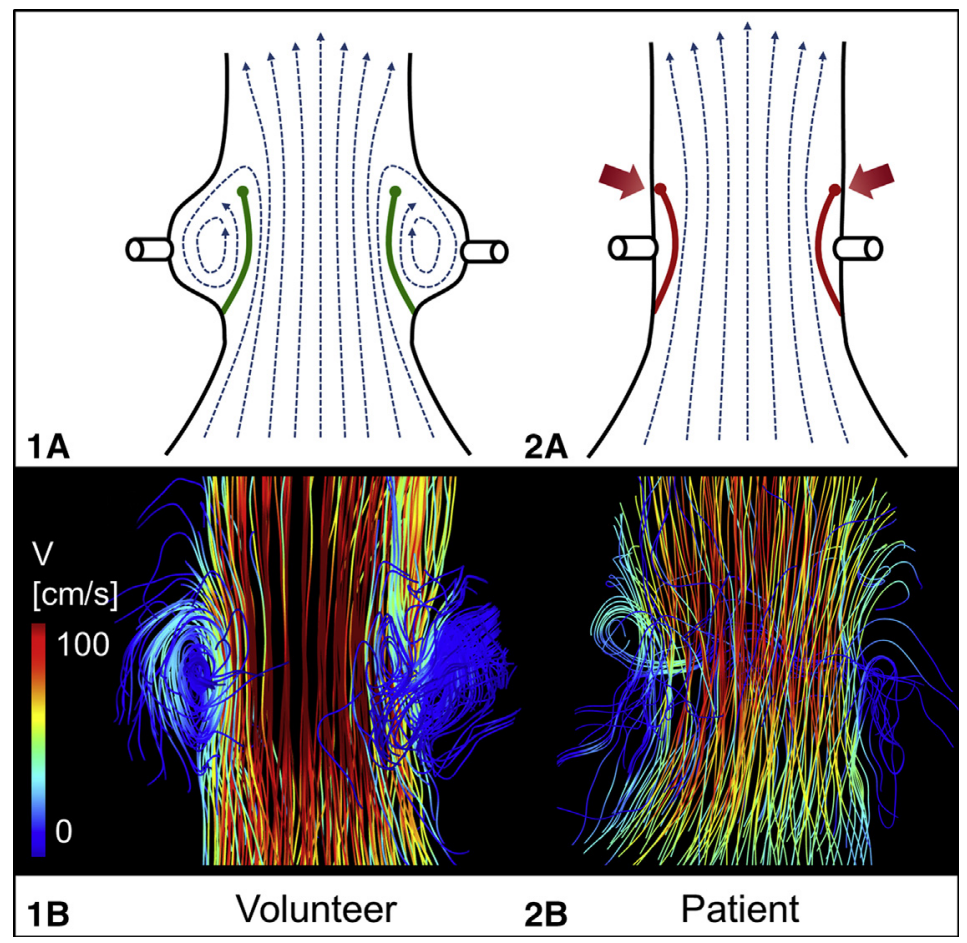

FIGURE 1. Aortic root hemodynamics in healthy volunteers and patients without sinuses of Valsalva. Schematic view (A) and 4-dimensional flow magnetic resonance imaging streamline visualization (B) of aortic root hemodynamics in a volunteer with sinuses of Valsalva ( $1 A$ and $1 B$ ). At peak systole, sinus vortices form behind the opened valve leaflets in the aortic sinuses. The valve leaflets do not contact the aortic wall because they are stabilized by the vortices. In a straight graft without aortic sinuses $(2 A$ and $2 B)$, there is no place for proper vortex formation behind the opened valve leaflets and the valve leaflets collide with the prosthesis wall (red arrows). For corresponding videos, please refer to Videos 1 and 2. 


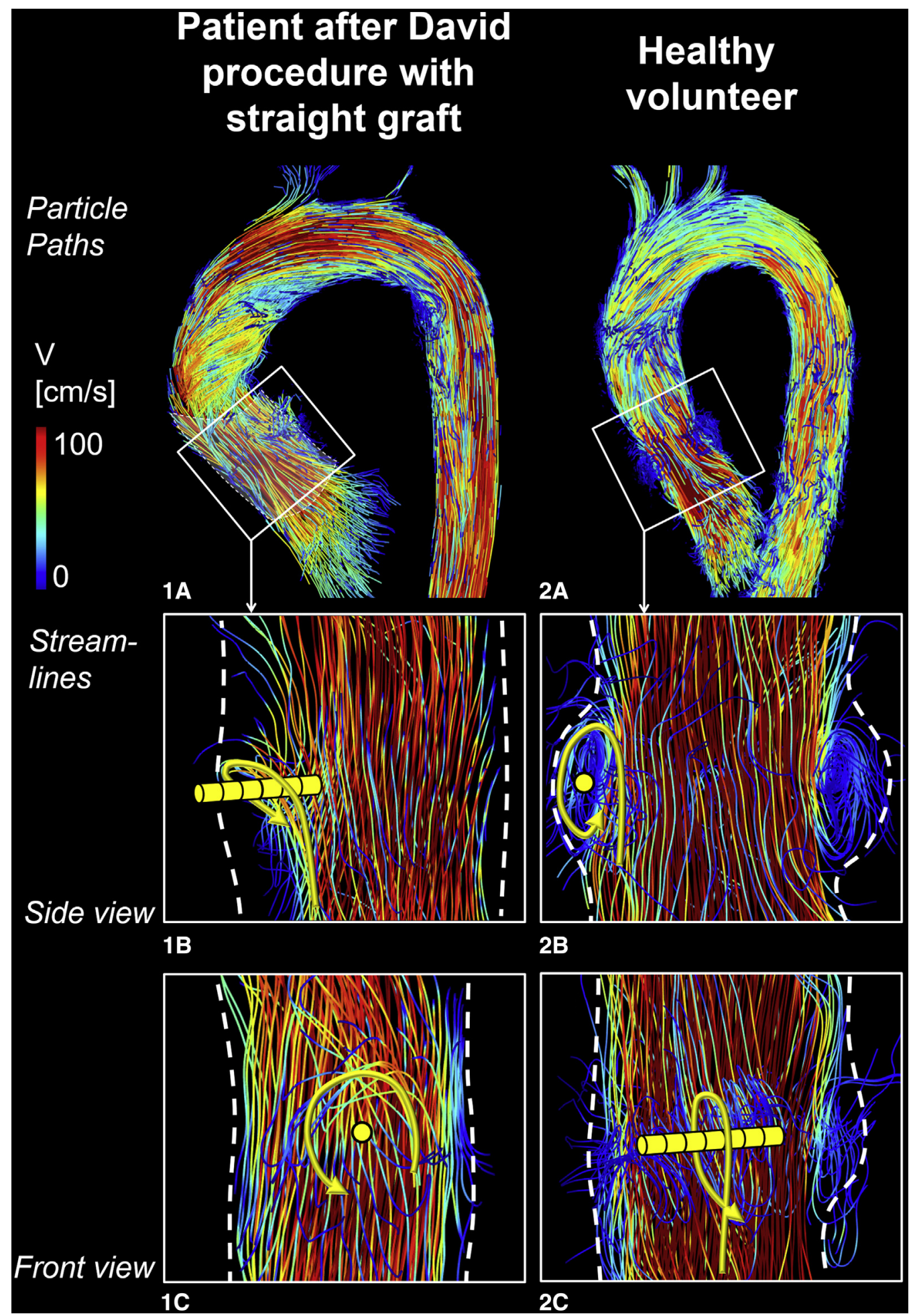

FIGURE 2. Sinus vortices in a patient after the David procedure (male, aged 41 years) and in a healthy volunteer (female, aged 53 years). Hemodynamics in the thoracic aorta visualized by particle paths color-coded to the flow velocity (top row). Side view (middle row) and front view (bottom row) of a sinus vortex in the right-coronary sinus displayed as instantaneous streamlines. The patient's sinus vortex is small and malrotated 90 degrees along the vessel axis in comparison with the volunteer's vortex. Dashed lines emphasize the vessel wall. Yellow arrows underline vortex direction. Rotation axis is marked as yellow bar or dot, depending on viewing angle. For corresponding videos please refer to Videos 3 and 4 . 


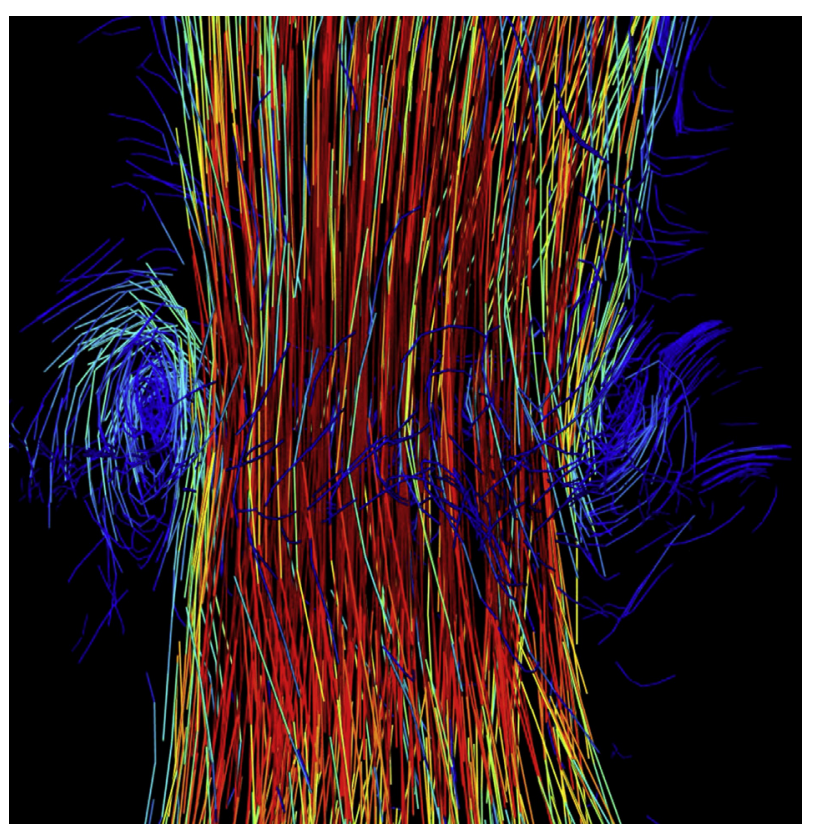

VIDEO 1. Aortic root hemodynamics in a healthy volunteer. Fourdimensional flow magnetic resonance imaging particle path visualization of aortic root hemodynamics in a volunteer with sinuses of Valsalva (female, aged 25 years). At peak systole, sinus vortices form behind the opened valve leaflets in the aortic sinuses. Video available at: http:// www.jtcvsonline.org/article/S0022-5223(17)30264-7/addons.

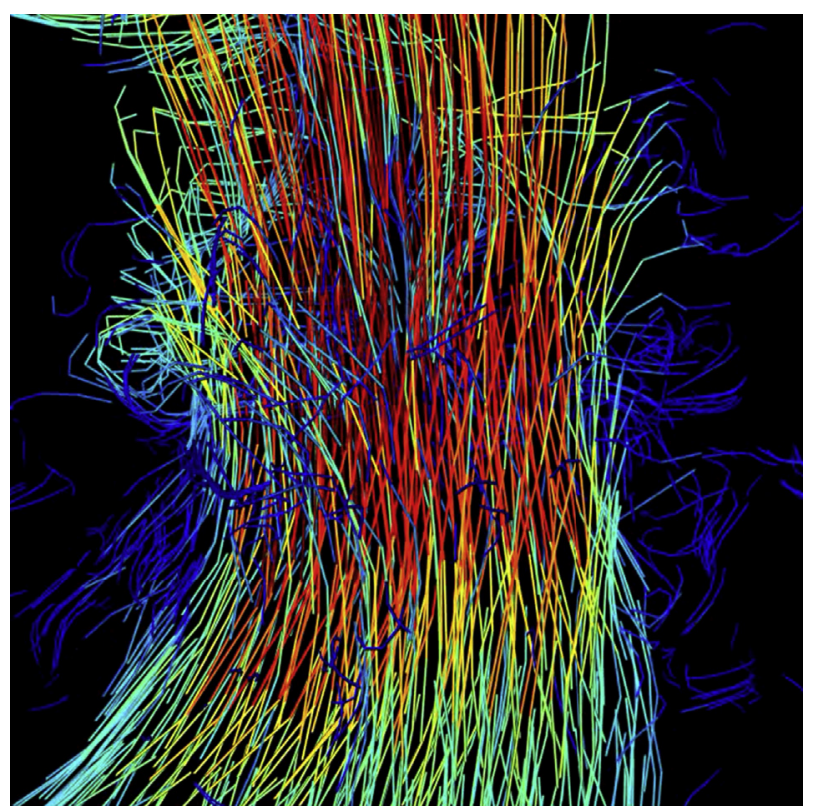

VIDEO 2. Aortic root hemodynamics in a patient after straight graft root replacement. Four-dimensional flow magnetic resonance imaging particle path visualization of aortic root hemodynamics in a patient after David procedure with a straight graft (male, aged 66 years). In a straight graft without aortic sinuses, there is no place for proper vortex formation behind the opened valve leaflets. Video available at: http://www.jtcvsonline.org/ article/S0022-5223(17)30264-7/addons.

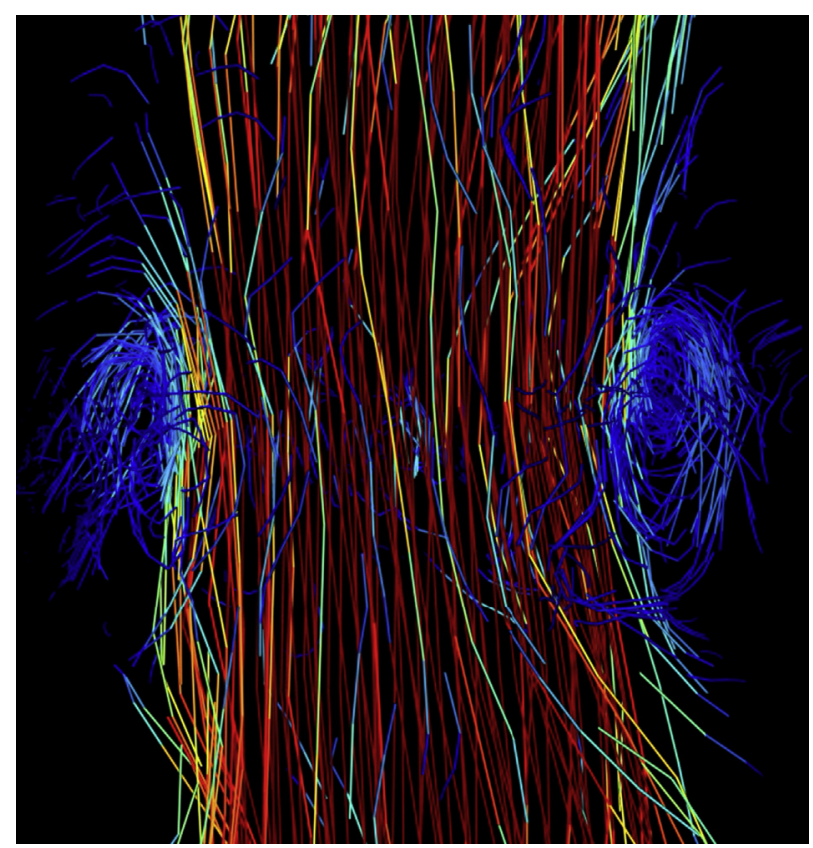

VIDEO 3. Sinus vortices in a healthy volunteer. Four-dimensional flow magnetic resonance imaging particle path visualization of aortic root hemodynamics in a volunteer (female, aged 53 years). At peak systole, sinus vortices form behind the opened valve leaflets in the aortic sinuses with their rotation axis parallel to the vessel wall in side view. Video available at: http://www.jtcvsonline.org/article/S0022-5223(17)30264-7/addons.

consequences for aortic valve function. Physiologically, sinus vortices develop behind the opened valve leaflets ${ }^{2}$ (Figure $1, I A$ and $I B$ ). They are attributed to minimizing leaflet stress ${ }^{3}$ and transvalvular pressure gradients, ${ }^{4}$ and to stabilizing leaflets in the open position. ${ }^{5}$ Depriving the patient of the sinuses may have consequences not yet fully recognized.

Three patients after David procedure with straight grafts were examined with an electrocardiogram-synchronized blood flow-sensitive magnetic resonance imaging sequence (4-dimensional flow magnetic resonance imaging [4D Flow MRI]) to measure time-resolved velocity information of every voxel within the aorta. Hemodynamics were analyzed with GTFlow (GyroTools GmbH, Winterthur, Switzerland) using color-coded techniques to visualize the acquired velocity information.

Small systolic vortices were detected behind the valve leaflets except for the noncoronary leaflet of 2 patients (Figure 1, $2 A$ and $2 B$ ). All vortices had a rotation axis orthogonal to the prosthesis wall. This finding contrasts the amount and direction of sinus vortices in a previously described study with 12 volunteers exhibiting sinus vortices with a rotation axis parallel to the vessel wall. ${ }^{6}$ Consequently, the presented patient's vortices were rotated approximately $90^{\circ}$ along the 


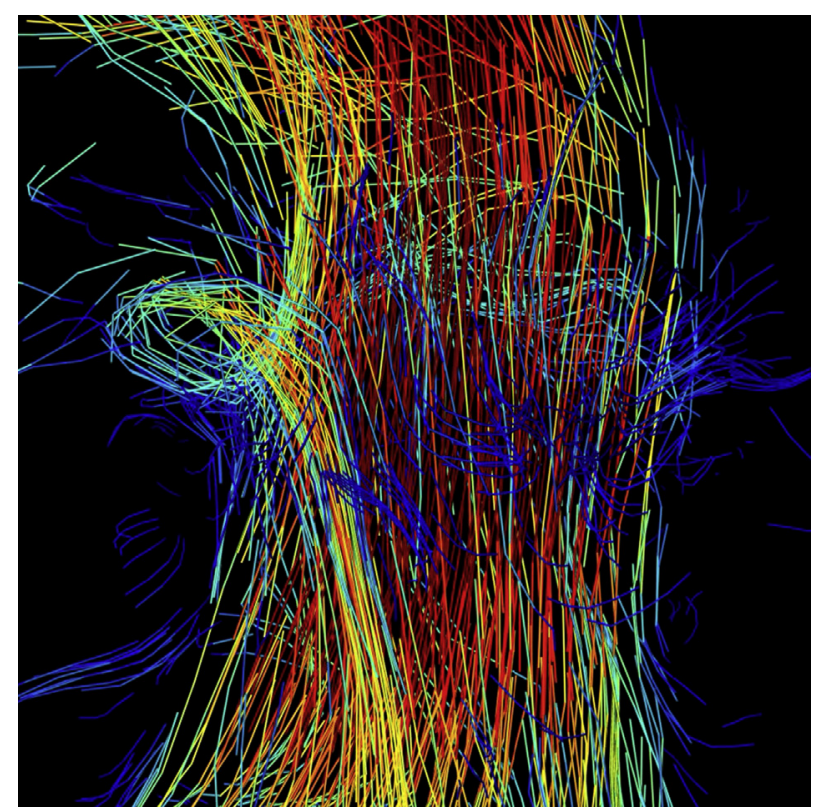

VIDEO 4. Sinus vortices in a patient after the David procedure. Fourdimensional flow magnetic resonance imaging particle path visualization of aortic root hemodynamics in a patient after the David procedure with a straight graft (male, aged 41 years). In a straight graft without aortic sinuses, there is no place for proper vortex formation behind the opened valve leaflets. The patient's sinus vortex is small with a rotation axis orthogonal to the prosthesis wall in side view. Thus, the vortex is malrotated $90^{\circ}$ along the vessel axis in comparison with the volunteer's vortex. Video available at: http://www.jtcvsonline.org/article/S0022-5223(17)30264-7/ addons.

vessel axis compared with the healthy volunteer's vortices (Figure 2).
This first description of malrotated vortices in straight grafts further substantiates previous echocardiographic results that revealed systolic contact of the valve leaflets with the prosthesis wall ${ }^{4}$ (Figure 1,2A) and higher leaflet stress ${ }^{3}$ in straight grafts. Initial follow-up suggests a slowly progressing deterioration of valve function. ${ }^{7}$ Thus, it may be hypothesized that the absence of sinuses relates to altered valve function through deformed sinus vortices. Therefore, the results in anatomically shaped sinus prostheses with nearphysiologic flow patterns in the aortic root previously presented in the Journal ${ }^{6}$ should trigger further comparative and follow-up studies in different aortic root replacement techniques.

\section{References}

1. David TE, Feindel CM. An aortic valve-sparing operation for patients with aortic incompetence and aneurysm of the ascending aorta. J Thorac Cardiovasc Surg. 1992;103:617-22.

2. Bellhouse BJ, Bellhouse FH. Mechanism of closure of the aortic valve. Nature 1968;217:86-7.

3. Grande-Allen KJ, Cochran RP, Reinhall PG, Kunzelman KS. Re-creation of sinuses is important for sparing the aortic valve: a finite element study. J Thorad Cardiovasc Surg. 2000;119:753-63.

4. Erasmi A, Sievers HH, Scharfschwerdt M, Eckel T, Misfeld M. In vitro hydrodynamics, cusp-bending deformation, and root distensibility for different types of aortic valve-sparing operations: remodeling, sinus prosthesis, and reimplantation. J Thorac Cardiovasc Surg. 2005;130:1044-9.

5. Caro CG, Pedley TJ, Schroter RC, Seed WA. The Mechanics of the Circulation. Cambridge: Cambridge University Press; 2012.

6. Oechtering TH, Hons CF, Sieren M, Hunold P, Hennemuth A, Huellebrand M, et al. Time-resolved 3-dimensional magnetic resonance phase contrast imaging (4D Flow MRI) analysis of hemodynamics in valve-sparing aortic root repair with an anatomically shaped sinus prosthesis. J Thorac Cardiovasc Surg. 2016; 152:418-27.e1.

7. Charitos EI, Stierle U, Tietze C, Hanke T, Karluss A, Misfeld M, et al. Clinical outcomes and lessons learned with aortic valve repair in 508 patients. J Heart Valve Dis. 2014;23:550-7. 